\title{
Alteration of Monoamine Receptor Activity and Glucose Metabolism in Pediatric Patients with Anticonvulsant-Induced Cognitive Impairment
}

\author{
Yuankai Zhu*1-4, Jianhua Feng*5, Jianfeng Ji*1-4, Haifeng Hou ${ }^{1-4}$, Lin Chen ${ }^{1-4}$, Shuang $\mathrm{Wu}^{1-4}$, Qing Liu ${ }^{1-4}$, \\ Qiong Yao ${ }^{1-4}$, Peizhen $\mathrm{Du}^{1-4}$, Kai Zhang ${ }^{1-4}$, Qing Chen ${ }^{1-4}$, Zexin Chen ${ }^{6}$, Hong Zhang ${ }^{1-4}$, and Mei Tian ${ }^{1-4}$ \\ ${ }^{I}$ Department of Nuclear Medicine, The Second Hospital of Zhejiang University School of Medicine, Hangzhou, China; \\ ${ }^{2}$ Zhejiang University Medical PET Centre, Zhejiang University, Hangzhou, China; ${ }^{3}$ Institute of Nuclear Medicine and \\ Molecular Imaging, Zhejiang University, Hangzhou, China; ${ }^{4}$ Key Laboratory of Medical Molecular Imaging of Zhejiang \\ Province, Hangzhou, China; ${ }^{5}$ Department of Paediatrics, The Second Hospital of Zhejiang University School of Medicine, \\ Hangzhou, China; and ${ }^{6}$ Department of Clinical Epidemiology \& Biostatistics. The Second Hospital of Zhejiang University \\ School of Medicine, Hangzhou, China
}

\begin{abstract}
A landmark study from the Institute of Medicine reported that the assessment of cognitive difficulties in children with epilepsy is timely and imperative. Anticonvulsant-induced cognitive impairment could influence the quality of life more than seizure itself in patients. Although the monoaminergic system is involved in the regulation of cognitive process, its role in anticonvulsant-induced cognitive impairment remains unclear. Methods: To explore in vivo monoamine receptor binding activity in patients with anticonvulsantinduced cognitive impairment, each patient underwent PET imaging with both monoamine receptor binding agent ${ }^{11} \mathrm{C}-\mathrm{N}$-methylspiperone and glucose metabolic agent ${ }^{18} \mathrm{~F}-\mathrm{FDG}$. Tests of intelligence quotient $(I Q)$, including verbal IQ $(\mathrm{VIQ})$, performance IQ (PIQ), and full-scale IQ (FSIQ), were performed in each patient. Results: Compared with the patients with monotherapy, patients with polytherapy had significantly lower VIQ, PIQ, and FSIQ $(P<0.01$ in each comparison), as well as significantly lower monoamine receptor activities detected in the caudate nucleus, prefrontal cortex, dorsal anterior cingulate cortex, and amygdale $(P<0.05$ in each comparison). However, regarding the glucose metabolism, there was no significant difference found in patients with monotherapy or polytherapy $(P>0.05)$. Conclusion: Monoamine receptor PET imaging could be a promising in vivo imaging biomarker for mapping anticonvulsant-induced cognitive impairment.
\end{abstract}

Key Words: epilepsy; cognition; anticonvulsant; monoamine receptor; positron emission tomography (PET)

J Nucl Med 2017; 58:1490-1497

DOI: 10.2967/jnumed.116.189290

\section{A}

landmark study from the Institute of Medicine reports that the chance of developing epilepsy at some point in our lives is 1 in 26 and recommends that the assessment of cognitive difficulties in

Received Dec. 29, 2016; revision accepted Feb. 27, 2017.

For correspondence or reprints contact: Mei Tian, Department of Nuclear Medicine, The Second Hospital of Zhejiang University, 88 Jiefang Rd., Hangzhou, Zhejiang 310009, China.

E-mail: meitian@zju.edu.cn

${ }^{*}$ Contributed equally to this work.

Published online Mar. 16, 2017.

COPYRIGHT (C) 2017 by the Society of Nuclear Medicine and Molecular Imaging. children with epilepsy is timely and imperative (1). Cognitive impairment is the most common side effect of anticonvulsants, and the risk may accumulate when anticonvulsants are combined (2). Particularly, the developing brains of pediatric patients appear to be particularly sensitive to the cognitive adverse effects of anticonvulsants due to the age-related pharmacokinetic and pharmacodynamic features (3).

PET is ideally suited for monitoring cell $/$ molecular events early in the course of a disease, as well as during pharmacologic or radiation therapy (4). Among PET radioligands for mapping postsynaptic monoamine receptors, ${ }^{11} \mathrm{C}$-labeled raclopride $\left({ }^{11} \mathrm{C}\right.$ raclopride) is the most often used as a standard $\mathrm{D}_{2}$ dopamine receptor $\left(\mathrm{D}_{2} \mathrm{DR}\right)$ ligand (5). However, because the binding of ${ }^{11} \mathrm{C}$-raclopride to the $\mathrm{D}_{2} \mathrm{DR}$ in the striatum is competitive with that of endogenous dopamine and reversible in the time frame of a PET scan, it could not reflect the actual changes of postsynaptic receptor level. In contrast, the binding of ${ }^{11} \mathrm{C}-\mathrm{N}$-methylspiperone $\left({ }^{11} \mathrm{C}-\mathrm{NMSP}\right.$; as a $\mathrm{D}_{2} \mathrm{DR}$ and 5-hydroxytryptamine receptor $2 \mathrm{~A}\left[5-\mathrm{HT}_{2 \mathrm{~A}} \mathrm{R}\right]$ antagonist, not competitive with that of endogenous dopamine $(5,6)$ and serotonin (7)) can directly reflect postsynaptic monoamine receptor levels more accurately than ${ }^{11} \mathrm{C}$ raclopride. Although the monoaminergic system is involved in the regulation of cognitive process, its role in anticonvulsant-induced cognitive impairment remains unclear $(8,9)$. Therefore, we performed ${ }^{11} \mathrm{C}$-NMSP PET studies to examine the alteration of monoamine receptor activity in pediatric patients with anticonvulsant-induced cognitive impairment.

\section{MATERIALS AND METHODS}

\section{Subjects}

Twenty-five patients ( 13 males; mean age \pm SD, $13.7 \pm 2.0$ y) diagnosed with epilepsy (10) were enrolled in this study (Table 1). Sixteen right-handed healthy control participants ( 9 males; mean age $\pm \mathrm{SD}, 21.6 \pm 1.3 \mathrm{y}$ ) with no history of neuropsychiatric disorders or receiving psychoactive drugs were included. The Institutional Review Board approved this study (ChiCTR-DDD-15007423), and written informed consent was obtained from each participant.

The patient inclusion criteria were as follows: clinical and electroencephalogram findings indicative of focal seizure, age $10-18 \mathrm{y}$, receiving at least 1 anticonvulsant and without alteration of its dosage in the 3 mo before this study, without developmental 
TABLE 1

Clinical Characteristics and Comparisons Between Mono- and Polytherapy Groups

\begin{tabular}{lccc}
\hline & \multicolumn{3}{c}{ Anticonvulsant } \\
\cline { 2 - 3 } Clinical characteristic & $\begin{array}{c}\text { Monotherapy } \\
(n=12)\end{array}$ & $\begin{array}{c}\text { Polytherapy } \\
(n=13)\end{array}$ & $P$ \\
\hline Sex & & & \\
\hline Female/male & $7 / 5$ & $5 / 8$ & 0.434 \\
\hline Age (y) & $13.3 \pm 1.9$ & $14.1 \pm 2.1$ & 0.302 \\
\hline Age of onset (y) & $7.6 \pm 3.0$ & $8.8 \pm 1.9$ & 0.250 \\
\hline Duration of epilepsy (y) & $5.7 \pm 2.9$ & $5.3 \pm 2.2$ & 0.749 \\
\hline IQ & & & \\
\hline Verbal & $105.6 \pm 17.5$ & $81.9 \pm 19.6$ & 0.004 \\
\hline Performance & $115.3 \pm 12.7$ & $86.9 \pm 25.5$ & 0.002 \\
\hline Full-scale & $111.5 \pm 16.7$ & $83.4 \pm 22.6$ & 0.002 \\
\hline MRI & & & \\
\hline Abnormal/normal & $2 / 10$ & $2 / 11$ & 1.000 \\
\hline Type of epilepsy & & & 1.000 \\
\hline RE & 6 & 7 & \\
\hline TLE & 5 & 5 & \\
\hline Frontal lobe epilepsy & 1 & 1 & \\
\hline Seizure activity & & & 0.041 \\
\hline Resistance/remission & $10 / 2$ & $5 / 8$ \\
\hline
\end{tabular}

disabilities, without history of addictions or other psychiatric illnesses, and right-handedness. Patients were excluded if they had identified epileptic encephalopathies or had comorbidities including depression, anxiety, and attention deficit hyperactivity disorder rather than cognitive impairment. Other exclusion criteria were diagnosis of tuberous sclerosis, tumors, or major hemispheric deformity (such as hemimegalencephaly and porencephaly), becuase these lesions preclude the normalization of PET images.

Each patient underwent at least 1 prolonged $(24 \mathrm{~h})$ electroencephalogram monitoring to confirm the localization of the epileptic foci. Video-electroencephalography was performed in 5 patients who presented difficulties in lesion localization. Tests of intelligence quotient (IQ), including verbal IQ (VIQ), performance IQ (PIQ), and full-scale IQ (FSIQ), were performed using the Chinese version of the Wechsler Intelligence Scale for Children (C-WISC) or Adults (C-WISA).

\section{PET Imaging with ${ }^{11} \mathrm{C}-\mathrm{NMSP}$ and ${ }^{18} \mathrm{~F}-\mathrm{FDG}$}

The PET imaging studies were done using a clinical PET/CT scanner (Biograph mCT; Siemens Medical Solutions). Dynamic scans were obtained at $0-40 \mathrm{~min}$ after a bolus intravenous injection of ${ }^{11} \mathrm{C}$ NMSP $(3.7 \mathrm{MBq} / \mathrm{kg})(11)$. A 5 -min static brain scan was acquired at $40 \mathrm{~min}$ after injection of ${ }^{18} \mathrm{~F}-\mathrm{FDG}(3.7 \mathrm{MBq} / \mathrm{kg}$ ) (12). Interictal PET studies were performed in patients at least $24 \mathrm{~h}$ after the last clinical seizure. A pediatric physician carefully monitored each subject during the entire PET imaging study.

\section{PET Data Analysis}

Regions of interest were drawn on both hemispheres in the stereotactically normalized images. Voxel-based analysis of asymmetry index (AI) was conducted according to the previous study using statistical parametric mapping (SPM8) (13). Briefly, AI images were calculated at each voxel according to the following formula: $\mathrm{AI}=($ left - right $) /([$ left +
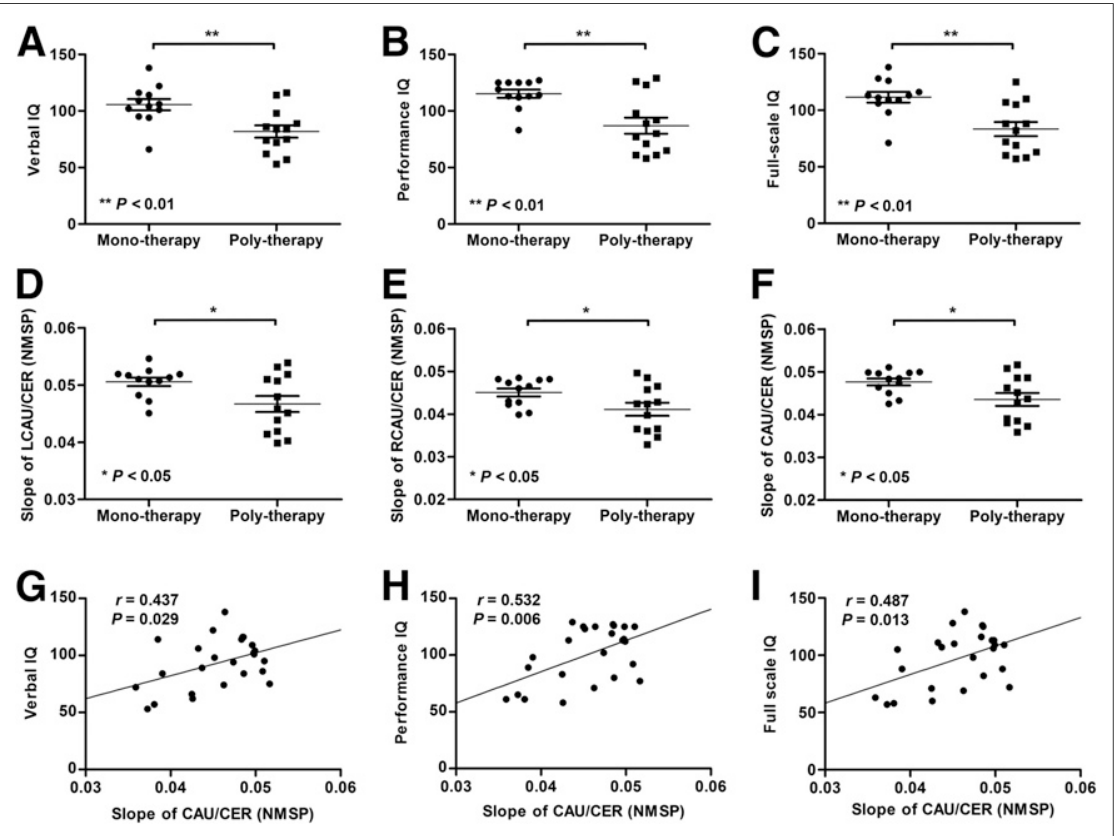

FIGURE 1. Anticonvulsant-induced cognitive impairment and alteration of monoamine receptor activity in caudate nucleus. (A-C) VIQ, PIQ, and FSIQ were significantly lower in patients with polytherapy compared with those with monotherapy ( $P<0.01$ in each comparison). (D-F) ${ }^{11} \mathrm{C}$ NMSP bindings in left, right, or entire caudate nucleus were significantly lower in patients with polytherapy than in those with monotherapy $(P<0.05$ in each comparison). (G-l) VIQ, PIQ, and FSIQ were significantly positively correlated with ${ }^{11} \mathrm{C}$-NMSP binding in caudate nucleus $(r=$ $0.437,0.532$, and 0.487 , respectively, $P<0.05$ in each comparison). right $] / 2)$. In the case of the patients with epileptic foci in the right hemisphere, the orientation of AI images were flipped along to the sagittal plane. AI images of patients were then compared with the controls, using the 2-sample $t$ test of the SPM software package, with an ANCOVA by each subject. Decreased or increased results were regarded as statistically significant if the uncorrected $P$ value was under $0.001\left({ }^{18} \mathrm{~F}-\mathrm{FDG}\right)$ or $0.01\left({ }^{11} \mathrm{C}\right.$ NMSP), respectively, with cluster level above 100 voxels.

\section{Statistical Analysis}

The results are presented as mean \pm SD. All data were analyzed by IBM SPSS Statistics (version 20.0; SPSS/IBM). Analysis of correlation was performed using Pearson correlation coefficients. Group differences in sex, age, age of onset, duration of epilepsy, MRI abnormality, epilepsy type, and IQ were tested using the 2-sample $t$ test or Fisher exact test as appropriate. Stepwise multivariate linear regression analysis was conducted to determine the independent contribution of individual variables (sex, age, age of onset, duration of epilepsy, time since last seizure, seizure frequency, lateralization of epileptic foci, epilepsy type, MRI abnormality, and combination of anticonvulsant) to the IQ or 
radiotracer. A $P$ value of less than 0.05 was considered statistically significant.

\section{RESULTS}

The clinical characteristics of the patients are summarized in Table 1. Twelve patients received 1 type of anticonvulsant, 12 patients received 2 types, and 1 patient required treatment with 3 types. All the patients were tested by C-WISC, except that 2 patients, aged 17.5 and $17.7 \mathrm{y}$, were tested by C-WISA. The most frequently used drug was oxcarbazepine (OXC; 16 patients), followed by topiramate (TPM; 9 patients), lamotrigine (LTG; 6 patients), levetiracetam (LEV; 4 patients), valproate (VPA; 3 patients), and clonazepam (CZP, 1 patient). The VIQ (Fig. 1A), PIQ (Fig. 1B), and FSIQ (Fig. 1C) in patients with monotherapy were significantly higher than those with polytherapy $(P<0.01$ in each comparison). However, the VIQ, PIQ, and FSIQ were not influenced by the sex $(P=0.711,0.934$, and 0.879 , respectively), age ( $P=0.812,0.948$, and 0.906 , respectively), age of onset ( $P=0.856,0.757$, and 0.802 , respectively), duration of epilepsy ( $P=0.881,0.857$, and 0.837 , respectively), time since last seizure $(P=0.534,0.284$, and 0.450 , respectively), seizure frequency $(P=0.348,0.338$, and 0.424 , respectively), lateralization of epileptic foci $(P=0.289,0.889$, and 0.482 , respectively), epilepsy type $(P=0.535,0.847$, and $P=0.650$, respectively), and MRI abnormality $(P=0.884,0.638$, and 0.769 , respectively).

To determine the independent contribution of individual variables (sex, age, age of onset, duration of epilepsy, time since last seizure, seizure frequency, lateralization of epileptic foci, epilepsy type, MRI abnormality, and combination of anticonvulsant) to the IQ, stepwise multivariate linear regression analysis was conducted. Only the combination of anticonvulsant (mono- vs. polytherapy) was found as the significant contributing variable to FSIQ $(\mathrm{B}=-28.1$; SEM $=8.0 ; P=0.002$ ).

\section{Monoamine Receptor Activity: Between-Group Differences (Monotherapy Versus Polytherapy)}

${ }^{11} \mathrm{C}-\mathrm{NMSP}$ bindings in the left (Fig. 1D), right (Fig. 1E), or entire (Fig. 1F) caudate nucleus, were significantly lower in patients with polytherapy than in those with monotherapy $(P<0.05$ in each comparison). No significant difference of ${ }^{11} \mathrm{C}-\mathrm{NMSP}$ binding was found between the 2 groups either in the putamen or in the pallidum $(P=0.085$ and 0.920 , respectively). Compared with
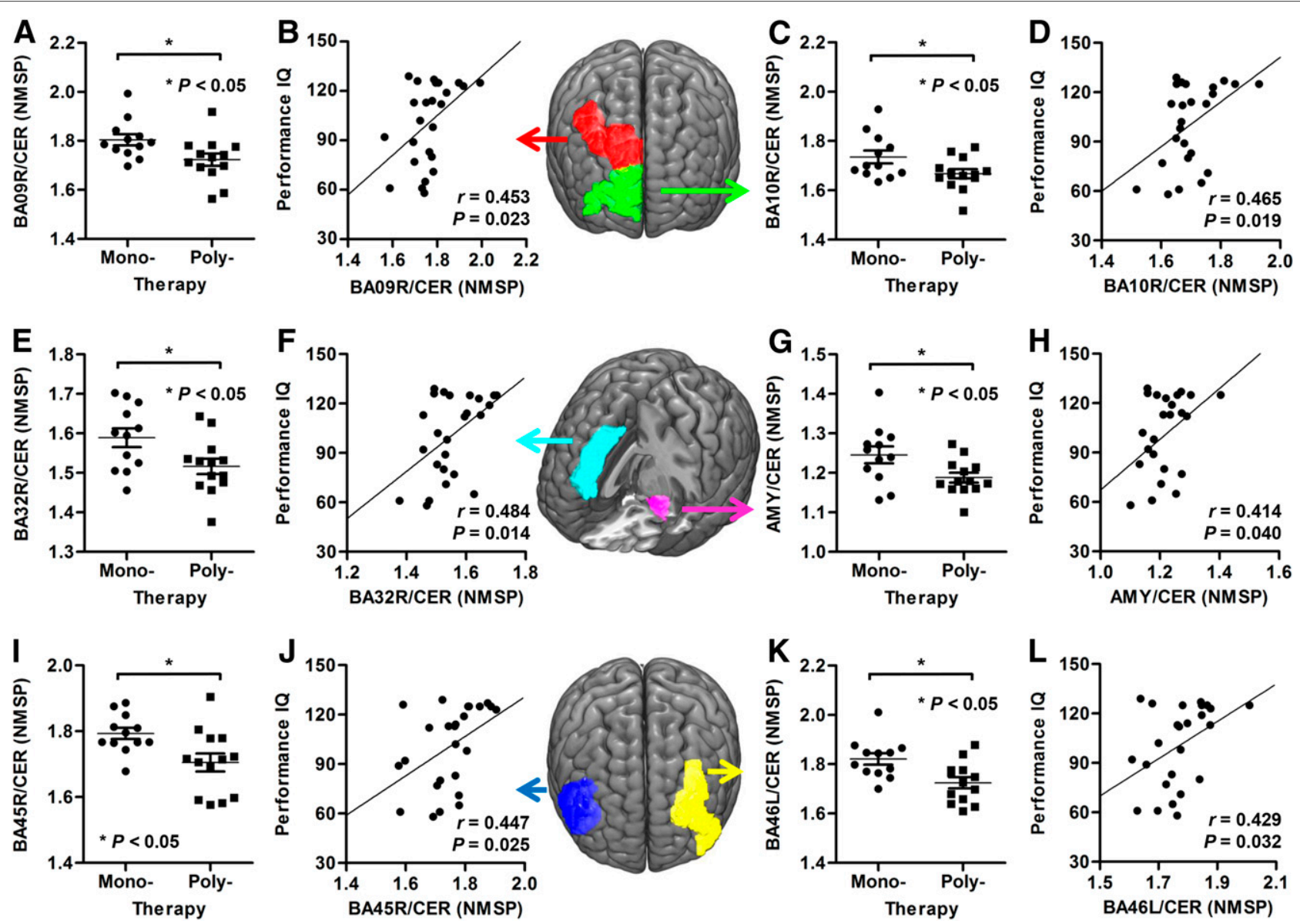

FIGURE 2. Anticonvulsant-induced cognitive impairment and alteration of monoamine receptor activity in cortex. (A, C, E, G, I, and K) Patients with polytherapy were detected with significantly lower ${ }^{11} \mathrm{C}-\mathrm{NMSP}$ bindings than those with monotherapy in BA9R, BA10R, BA32R, BA45R, BA46L, and amygdala $\left(P<0.05\right.$ in each comparison). (B, D, F, H, J, and L) ${ }^{11} \mathrm{C}-\mathrm{NMSP}$ bindings in BA9R, BA10R, BA32R, BA45R, BA46L, and amygdala were significantly positively correlated with $\mathrm{PIQ}(r=0.453,0.465,0.484,0.447,0.429$, and 0.414 , respectively, $P<0.05$ in each comparison). 
TABLE 2

Results of Multivariate Linear Regression: Independent Contributions to Monoamine Receptor Activity (Mono- Versus Polytherapy)

\begin{tabular}{lccc}
\hline \multicolumn{1}{c}{ Brain region } & $\beta$ & SEM & $P$ \\
\hline Caudate nucleus & -0.004 & 0.002 & 0.029 \\
Amygdala & -0.058 & 0.024 & 0.026 \\
BA9R & -0.080 & 0.034 & 0.027 \\
BA10R & -0.068 & 0.032 & 0.041 \\
BA32R & -0.073 & 0.031 & 0.026 \\
BA45R & -0.088 & 0.033 & 0.013 \\
BA46L & -0.096 & 0.033 & 0.007 \\
\hline
\end{tabular}

those with monotherapy, patients with polytherapy were detected with significantly lower ${ }^{11} \mathrm{C}$-NMSP bindings in the right Broadman area 9 (BA9R; Fig. 2A), right Broadman area 10 (BA10R; Fig. 2C), right Broadman area 32 (BA32R; Fig. 2E), right
Broadman area 45 (BA45R; Fig. 2I), left Broadman area 46 (BA46L; Fig. 2K), and amygdala (Fig. 2G) $(P<0.05$ in each comparison).

In these brain regions, a combination of anticonvulsants was the only significant contributing variable to ${ }^{11} \mathrm{C}-\mathrm{NMSP}$ binding using stepwise multivariate linear regression analysis (Table $2, P<0.05$ in each region). However, regarding the glucose metabolism, there was no significant difference found in patients with monotherapy or polytherapy $(P>0.05)$.

\section{Correlation Between Monoamine Receptor Activity and Cognitive Function}

Regarding the relationship between regional ${ }^{11} \mathrm{C}-\mathrm{NMSP}$ binding and VIQ (Fig. 1G), PIQ (Fig. 1H), or FSIQ (Fig. 1I), significantly positive correlations were found in the caudate nucleus $(r=0.437$, 0.532 , and 0.487 , respectively, $P<0.05$ in each comparison), but neither in the putamen $(P=0.262,0.407$, and 0.330 , respectively), nor in the pallidum $(P=0.305,0.853$, and 0.520 , respectively). The ${ }^{11} \mathrm{C}-\mathrm{NMSP}$ bindings in BA9R (Fig. 2B), BA10R (Fig. 2D), BA32R (Fig. 2F), BA45R (Fig. 2J), BA46L (Fig. 2L), and amygdala (Fig. 2H) were significantly positively correlated with PIQ $(r=$ $0.453,0.465,0.484,0.447,0.429$, and 0.414 , respectively, $P<0.05$ in each comparison), but not with VIQ $(P=0.122$, $0.075,0.129,0.377,0.064$, and 0.201 , respectively). The FSIQ was significantly positively correlated with the ${ }^{11} \mathrm{C}-\mathrm{NMSP}$ bindings in BA9R, BA10R, BA32R, and BA46L $(r=0.398,0.437,0.397$, and 0.420 , respectively, $P<0.05$ in each comparison).

\section{Correlation Between Monoamine Receptor Activity and Glucose Metabolism}

In patients with rolandic epilepsy (RE), SPM analysis revealed significant reductions of ${ }^{11} \mathrm{C}$-NMSP binding in the ipsilateral postcentral gyrus, superior temporal gyrus, lingual gyrus, and rolandic operculum $\left(P_{\text {uncorrected }}<0.01\right.$, cluster size $>$ 100; Fig. 3A; Table 3) and found significant hypometabolism in the ipsilateral rolandic operculum, superior temporal gyrus, postcentral gyrus, and hippocampal gyrus $\left(P_{\text {uncorrected }}<0.001\right.$, cluster size $>$ 100; Fig. 3A; Table 4). Decreased monoamine receptor binding and glucose metabolism were found overlapped in the rolandic operculum, postcentral gyrus, and superior temporal gyrus (Fig. 3A). The ${ }^{11} \mathrm{C}-\mathrm{NMSP}$ binding in the ipsilateral rolandic operculum was significantly positively correlated with glucose metabolism $(r=0.651, P<0.05$; Fig. 3C).

For the temporal lobe epilepsy (TLE) patients, significant reductions of ${ }^{11} \mathrm{C}$ NMSP binding were found in the ipsilateral parahippocampal gyrus and inferior and middle temporal gyri $\left(P_{\text {uncorrected }}<0.01\right.$, 
TABLE 3

Results of SPM Analysis on NMSP Binding (Epilepsy Patients Versus Control Group)

\begin{tabular}{|c|c|c|c|c|c|c|c|c|c|c|c|c|}
\hline \multirow[b]{3}{*}{ Region } & \multicolumn{4}{|c|}{ Cluster level } & \multicolumn{5}{|c|}{ Peak level } & & & \\
\hline & \multirow[b]{2}{*}{$\mathrm{K}_{\mathrm{E}}$} & \multirow{2}{*}{$\begin{array}{c}P \\
\text { (FWE- } \\
\text { corrected) }\end{array}$} & \multirow{2}{*}{$\begin{array}{c}P \\
\text { (FDR- } \\
\text { corrected) }\end{array}$} & \multirow{2}{*}{$\begin{array}{c}P \\
\text { (uncorrected) }\end{array}$} & \multirow{2}{*}{$\begin{array}{c}P \\
\text { (FWE- } \\
\text { corrected) }\end{array}$} & \multirow{2}{*}{$\begin{array}{c}P \\
\text { (FDR- } \\
\text { corrected) }\end{array}$} & \multirow[b]{2}{*}{$\mathrm{T}$} & \multirow[b]{2}{*}{ Z } & \multirow{2}{*}{$\begin{array}{c}P \\
\text { (uncorrected) }\end{array}$} & \multicolumn{3}{|c|}{ Coordinate } \\
\hline & & & & & & & & & & $x$ & $y$ & $\bar{z}$ \\
\hline \multicolumn{13}{|l|}{ RE vs. control } \\
\hline \multicolumn{13}{|l|}{ Decreased } \\
\hline \multirow[t]{2}{*}{ Postcentral } & 129 & 0.811 & 0.756 & 0.049 & 0.747 & 0.650 & 4.35 & 3.74 & 0.000 & -41 & -17 & 44 \\
\hline & & & & & 1.000 & 0.918 & 2.70 & 2.51 & 0.006 & -61 & -11 & 37 \\
\hline \multirow[t]{2}{*}{ Temporal_Sup } & 125 & 0.830 & 0.756 & 0.052 & 0.757 & 0.650 & 4.34 & 3.73 & 0.000 & -56 & -39 & 20 \\
\hline & & & & & 1.000 & 0.897 & 2.99 & 2.75 & 0.003 & -42 & -34 & 12 \\
\hline \multirow[t]{2}{*}{ Lingual } & 114 & 0.897 & 0.756 & 0.062 & 0.988 & 0.897 & 3.75 & 3.32 & 0.000 & -12 & -54 & -2 \\
\hline & & & & & 1.000 & 0.897 & 3.42 & 3.08 & 0.001 & -18 & -58 & 2 \\
\hline \multirow{3}{*}{$\begin{array}{l}\text { Rolandic_Oper/ } \\
\text { Temporal_Sup }\end{array}$} & 143 & 0.739 & 0.756 & 0.039 & 1.000 & 0.897 & 2.99 & 2.75 & 0.003 & -44 & -20 & 20 \\
\hline & & & & & 1.000 & 0.897 & 2.90 & 2.67 & 0.004 & -58 & -14 & 13 \\
\hline & & & & & 1.000 & 0.951 & 2.57 & 2.41 & 0.008 & -54 & -22 & 7 \\
\hline \multicolumn{13}{|l|}{ Increased } \\
\hline \multirow[t]{2}{*}{$\begin{array}{l}\text { Frontal_Sup_Medial/ } \\
\text { Frontal_Sup }\end{array}$} & 113 & 0.883 & 0.573 & 0.063 & 0.815 & 0.282 & 4.25 & 3.67 & 0.000 & -10 & 60 & 25 \\
\hline & & & & & 1.000 & 0.759 & 2.78 & 2.58 & 0.005 & -16 & 62 & 11 \\
\hline Frontal_Mid & 141 & 0.749 & 0.573 & 0.040 & 0.985 & 0.358 & 3.78 & 3.34 & 0.000 & -32 & 50 & 19 \\
\hline \multicolumn{13}{|l|}{ TLE vs. control } \\
\hline \multicolumn{13}{|l|}{ Decreased } \\
\hline \multirow{3}{*}{$\begin{array}{l}\text { Para-Hippocampal/ } \\
\text { Temporal_Inf }\end{array}$} & 194 & 0.442 & 0.325 & 0.016 & 0.701 & 0.656 & 4.55 & 3.81 & 0.000 & -28 & -34 & -12 \\
\hline & & & & & 1.000 & 0.695 & 3.54 & 3.13 & 0.001 & -38 & -38 & -12 \\
\hline & & & & & 1.000 & 0.695 & 3.34 & 2.98 & 0.001 & -30 & -24 & -18 \\
\hline \multirow{3}{*}{$\begin{array}{l}\text { Temporal_Mid/ } \\
\text { Temporal_Inf }\end{array}$} & 267 & 0.197 & 0.245 & 0.006 & 0.836 & 0.656 & 4.34 & 3.67 & 0.000 & -56 & -10 & -13 \\
\hline & & & & & 0.967 & 0.656 & 4.00 & 3.45 & 0.000 & -64 & -22 & -21 \\
\hline & & & & & 0.997 & 0.695 & 3.69 & 3.24 & 0.001 & -64 & -2 & -21 \\
\hline $\begin{array}{l}\text { Temporal_Mid/ } \\
\text { Temporal_Inf }\end{array}$ & 139 & 0.735 & 0.493 & 0.037 & 1.000 & 0.695 & 3.24 & 2.91 & 0.002 & -56 & -40 & -6 \\
\hline & & & & & 1.000 & 0.797 & 3.05 & 2.76 & 0.003 & -64 & -38 & -6 \\
\hline & & & & & 1.000 & 0.839 & 2.83 & 2.59 & 0.005 & -60 & -50 & -14 \\
\hline Increased & & & & & & & & & & & & \\
\hline Cingulum_Post/ & 101 & 0.918 & 0.672 & 0.070 & 0.677 & 0.718 & 4.59 & 3.83 & 0.000 & -9 & -36 & 32 \\
\hline & & & & & 0.984 & 0.718 & 3.89 & 3.37 & 0.000 & -1 & -40 & 36 \\
\hline Frontal_Sup_Orb/ & 148 & 0.684 & 0.672 & 0.032 & 0.961 & 0.718 & 4.03 & 3.47 & 0.000 & -16 & 60 & -11 \\
\hline & & & & & 0.999 & 0.718 & 3.63 & 3.19 & 0.001 & -42 & 50 & -3 \\
\hline & & & & & 1.000 & 0.771 & 3.24 & 2.91 & 0.002 & -28 & 58 & -13 \\
\hline
\end{tabular}

$\mathrm{K}_{\mathrm{E}}=$ cluster size; FWE = familywise error; FDR = false-discovery rate corrected; Sup = superior; Oper = operculum; Mid = middle; Inf = inferior; Post = posterior; Orb = orbital.

cluster size > 100; Fig. 3B; Table 3); in addition, hypometabolism presented in the ipsilateral inferior, middle, and superior temporal gyri; hippocampal gyrus; fusiform gyrus; and rolandic operculum $\left(P_{\text {uncorrected }}<0.001\right.$, cluster size $>100$; Fig. 3B; Table 4). Monoamine receptor dysfunction overlapped with the glucose metabolic abnormality in the inferior and middle temporal gyri (Fig. 3B). ${ }^{11} \mathrm{C}$ NMSP bindings were significantly positively correlated with glucose metabolism in the ipsilateral middle temporal gyrus $(r=$
0.756, $P<0.05$; Fig. 3D) and parahippocampal gyrus $(r=$ 0.855, $P<0.05$; Fig. 3E).

\section{DISCUSSION}

We report that cognitive performance significantly positively correlated with the ${ }^{11} \mathrm{C}-\mathrm{NMSP}$ binding in the caudate nucleus, prefrontal cortex (PFC), dorsal anterior cingulate cortex (dACC), 
TABLE 4

Results of SPM Analysis on ${ }^{18}$ F-FDG Uptake (Epilepsy Patients Versus Control Group)

\begin{tabular}{|c|c|c|c|c|c|c|c|c|c|c|c|c|}
\hline \multirow[b]{3}{*}{ Region } & \multicolumn{4}{|c|}{ Cluster level } & \multicolumn{5}{|c|}{ Peak level } & & & \\
\hline & \multirow[b]{2}{*}{$\mathrm{K}_{\mathrm{E}}$} & \multirow{2}{*}{$\begin{array}{c}P \\
\text { (FWE- } \\
\text { corrected) }\end{array}$} & \multirow{2}{*}{$\begin{array}{c}P \\
\text { (FDR- } \\
\text { corrected) }\end{array}$} & \multirow{2}{*}{$\begin{array}{c}P \\
\text { (uncorrected) }\end{array}$} & \multirow{2}{*}{$\begin{array}{c}P \\
(\text { FWE- } \\
\text { corrected) }\end{array}$} & \multirow{2}{*}{$\begin{array}{c}P \\
\text { (FDR- } \\
\text { corrected) }\end{array}$} & \multirow[b]{2}{*}{$\mathrm{T}$} & \multirow[b]{2}{*}{ Z } & \multirow{2}{*}{$\begin{array}{c}P \\
\text { (uncorrected) }\end{array}$} & \multicolumn{3}{|c|}{ Coordinate } \\
\hline & & & & & & & & & & $x$ & Y & $z$ \\
\hline \multicolumn{13}{|l|}{ RE vs. control } \\
\hline \multicolumn{13}{|l|}{ Decreased } \\
\hline $\begin{array}{l}\text { Rolandic_Oper/ } \\
\text { Temporal_Sup }\end{array}$ & 602 & 0.000 & 0.000 & 0.000 & 0.113 & 0.155 & 5.55 & 4.44 & 0.000 & -42 & -18 & 16 \\
\hline \multirow[t]{2}{*}{ Postcentral } & & & & & 0.135 & 0.155 & 5.47 & 4.39 & 0.000 & -50 & -42 & 22 \\
\hline & & & & & 0.148 & 0.155 & 5.42 & 4.37 & 0.000 & -52 & -20 & 22 \\
\hline Hippocampal & 108 & 0.041 & 0.063 & 0.006 & 0.349 & 0.230 & 4.94 & 4.09 & 0.000 & -26 & -26 & -8 \\
\hline \multicolumn{13}{|l|}{ Increased } \\
\hline Cingulum_Mid & 100 & 0.053 & 0.029 & 0.007 & 0.507 & 0.195 & 4.69 & 3.94 & 0.000 & -8 & -42 & 42 \\
\hline \multicolumn{13}{|l|}{ TLE vs. control } \\
\hline \multicolumn{13}{|l|}{ Decreased } \\
\hline \multirow[t]{3}{*}{$\begin{array}{l}\text { Temporal_Inf/ } \\
\text { Temporal_Mid }\end{array}$} & 231 & 0.002 & 0.003 & 0.000 & 0.216 & 0.316 & 5.38 & 4.25 & 0.000 & -58 & -28 & -18 \\
\hline & & & & & 0.424 & 0.341 & 4.95 & 4.01 & 0.000 & -62 & -44 & -6 \\
\hline & & & & & 0.982 & 0.759 & 3.86 & 3.33 & 0.000 & -64 & -16 & -18 \\
\hline Hippocampal & 128 & 0.024 & 0.025 & 0.003 & 0.261 & 0.316 & 5.27 & 4.19 & 0.000 & -28 & -24 & -8 \\
\hline Fusiform & 137 & 0.019 & 0.025 & 0.003 & 0.281 & 0.316 & 5.22 & 4.17 & 0.000 & -30 & -8 & -36 \\
\hline $\begin{array}{l}\text { Temporal_Sup/ } \\
\text { Rolandic_Oper }\end{array}$ & 366 & 0.000 & 0.000 & 0.000 & 0.308 & 0.316 & 5.16 & 4.13 & 0.000 & -56 & -12 & -2 \\
\hline \multirow[t]{2}{*}{ Temporal_Mid } & & & & & 0.430 & 0.341 & 4.94 & 4.01 & 0.000 & -40 & -30 & 22 \\
\hline & & & & & 0.612 & 0.380 & 4.66 & 3.84 & 0.000 & -64 & -30 & 4 \\
\hline \multicolumn{13}{|l|}{ Increased } \\
\hline Cingulum_Mid & 286 & 0.000 & 0.001 & 0.000 & 0.183 & 0.329 & 5.48 & 4.31 & 0.000 & -12 & -36 & 36 \\
\hline
\end{tabular}

$\mathrm{K}_{\mathrm{E}}=$ cluster size; FWE = familywise error; FDR = false-discovery rate corrected; Oper = operculum; Sup = superior; Mid = middle; Inf $=$ inferior.

and amygdale. This result suggests that polytherapy-induced cognitive impairment is related to the reduction of monoamine receptor activity. To the best of our knowledge, this is the first study on the neural correlate between anticonvulsant-induced cognitive impairment and the alteration of monoamine receptor activity or glucose metabolism detected by in vivo PET imaging with ${ }^{11} \mathrm{C}-\mathrm{NMSP}$ and ${ }^{18} \mathrm{~F}-\mathrm{FDG}$ in pediatric epilepsy.

Our study demonstrated that in patients with polytherapy, monoamine receptor activities decreased in the caudate nucleus, PFC (BA9R, BA10R, BA45R, and BA46L), dACC (BA32R), and amygdala. This is consistent with the preclinical study on anticonvulsant-induced impaired cognitive function in nonepileptic rats in which impaired cognition was found to be attributed to the increased hippocampal 5-HT levels for phenytoin (14), but to the decreased hippocampal dopamine levels for ethosuximide (15). However, phenytoin-associated elevated monoamine levels in the cortex and hippocampus could not justify the associated memory loss in a rat model of epilepsy (10). These discrepancies might be explained by the suggestion that increased or decreased release of monoamines depends on the concentration of anticonvulsants and the procedure used to evoke neurotransmitter release
(17). In adult TLE patients, no significant effects of anticonvulsants on $5-\mathrm{HT}_{1 \mathrm{~A}} \mathrm{R}$ binding were observed using ${ }^{18} \mathrm{~F}-\mathrm{FCWAY}$ PET imaging (18). The adverse effect of anticonvulsants on cognition could also be attributed to $\mathrm{Na}+$ channel blockade, enhanced GABAergic activity, or decrement in glutamate-mediated excitation (19). On the basis of these findings and our results, we speculated that the interaction between multiple anticonvulsants in ion channels and neurotransmitter receptors might aggravate the anticonvulsant-induced cognitive impairment. Because the research on the mechanism of anticonvulsant-induced cognitive impairment is still in its infancy, with many issues that need to be addressed, elucidation of its mechanism has been appraised as one of the epilepsy research priorities (20).

PET imaging in this study indicated that the cognitive performance (VIQ, PIQ, and FSIQ) significantly positively correlated with the ${ }^{11} \mathrm{C}-\mathrm{NMSP}$ binding in caudate nucleus. Accumulated evidence has shown that the dopaminergic system, especially $\mathrm{D}_{2} \mathrm{DR}$, is profoundly associated with cognition $(8,21)$. $\mathrm{D}_{2} \mathrm{DR}$ makes a specific contribution to hippocampus-based cognition by influencing the striatum and limbic system, and their interactions (21). Another important finding in our study was that 
${ }^{11} \mathrm{C}-\mathrm{NMSP}$ bindings in the PFC, dACC, and amygdala were positively correlated with PIQ. Apart from dopamine, 5-HT signaling at $5-\mathrm{HT}_{2 \mathrm{~A}} \mathrm{R}$ also has important effects on several behavioral and cognitive pathways, with the PFC as the central actor (9). In addition, activation of the $5-\mathrm{HT}_{2 \mathrm{~A}} \mathrm{R}$ in $\mathrm{PFC}$ has a modulatory effect on dopamine neurons, indicating that 5-HT can interact with other modulators of diverse cognitive processes (22). Though the amygdala lies at the center of much of our current thinking about emotion, its role is indeed quite broad and connected with cognitive functions according to amygdala-PFC interactions (23). Additionally, the dACC is also supposed to play a key role in fundamental cognitive processes, including motivation, decision making, and learning (24). Our data further highlighted the crucial role of $\mathrm{D}_{2} \mathrm{DR}$ and $5-\mathrm{HT}_{2 \mathrm{~A}} \mathrm{R}$ on cognitive function in pediatric patients with epilepsy.

In our current study, significant decreases in VIQ, PIQ, and FSIQ were found in patients with polytherapy compared with those with monotherapy. This finding is in line with a recent study that each additional drug increased the risk of cognitive side effects (2). Cessation of anticonvulsants was the strongest predictor of postoperative IQ increase, even after exclusion of patients with continuing seizures (25). Therefore, it is recommended that epilepsy patients should be treated with a single anticonvulsant wherever possible, and combination anticonvulsants should be considered only when attempts at a single anticonvulsant have not resulted in seizure freedom (26). Routine cognitive monitoring of anticonvulsants would be highly valuable to optimize outcomes and improve adherence to the prescribed medication (27). Apart from conventional IQ test, we suggested that ${ }^{11} \mathrm{C}$-NMSP PET imaging could be one potential evaluation approach for the cognitive monitoring in pediatric epilepsy.

${ }^{18}$ F-FDG PET imaging of brain glucose metabolism is a wellestablished and widely available technique for assessment of epilepsy, and the characteristic finding is a regional hypometabolism during the interictal period. Although hypometabolism has been ascribed to factors such as neuronal loss and diaschisis, its underlying neurobiology is not well understood (28). In the present study, deceased monoamine receptor activity and glucose metabolism were found overlapped in epileptic foci in patients with RE and TLE. The positive correlation between hypometabolism and the reduction of monoamine receptor binding in our study suggested that the abnormal cerebral metabolism might be associated with alterations in neurotransmitters and synaptic activity. The occurrence of epileptic seizures has been explained by an imbalance between excitatory (glutamatergic) and inhibitory (GABAergic) neurotransmission. Some other neurotransmitter systems are known to be involved in the epileptogenesis, including dopamine and 5-HT (29). PET studies revealed a reduction of $D_{2} R / D_{3} R$ binding in TLE and juvenile myoclonic epilepsy $(30,31)$ and reduction of 5$\mathrm{HT}_{1 \mathrm{~A}} \mathrm{R}$ binding ipsilateral to TLE foci (13). Our study further demonstrated that $5-\mathrm{HT}_{2 \mathrm{~A}} \mathrm{R}$ binding decreased both in $\mathrm{RE}$ and in TLE patients. This finding is inconsistent with the previous report (32). 5-HT has an anticonvulsant effect in epilepsy, and the $5-\mathrm{HT}_{2 \mathrm{~A}} \mathrm{Rs}$ appear to play a major role. However, further studies are needed to understand the role of $5-\mathrm{HT}_{2 \mathrm{~A}} \mathrm{R}$ in epilepsy and its modulation of other neurotransmitter systems.

It is important to acknowledge the potential limitation in this study. First, the baseline cognitive function before anticonvulsant therapy, usually $5 \mathrm{y}$ ago, was not considered in the current study. However, numerous studies have demonstrated that the cognitive impairment may cumulate when anticonvulsants are combined, regardless of the pretreatment assessment of cognitive function (2). Second, we did not find significant correlation between cognitive impairment and other clinical variables, including age of onset, duration of epilepsy, and seizure frequency, which might be due to the limited numbers in each category.

\section{CONCLUSION}

Our findings document that cognitive performance significantly positively correlated with the monoamine receptor binding in the caudate nucleus, PFC, dACC, and amygdale in pediatric epilepsy. These results indicated an important role of monoamine receptor dysfunction in anticonvulsant-induced cognitive impairment.

\section{DISCLOSURE}

This work is partly sponsored by grants from the National Key Research and Development Program of China (2016YFA0100900), National Key Basic Research Program of China (2013CB329506), National Science Foundation of China (NSFC) (no. 81425015, 81271601), and Zhejiang Provincial Natural Science Foundation of China (LR13H180001). No other potential conflict of interest relevant to this article was reported.

\section{REFERENCES}

1. Wilson SJ, Baxendale S, Barr W, et al. Indications and expectations for neuropsychological assessment in routine epilepsy care: report of the ILAE Neuropsychology Task Force, Diagnostic Methods Commission, 2013-2017. Epilepsia. 2015;56:674-681.

2. Witt JA, Elger CE, Helmstaedter C. Adverse cognitive effects of antiepileptic pharmacotherapy: each additional drug matters. Eur Neuropsychopharmacol. 2015;25:1954-1959.

3. Italiano D, Perucca E. Clinical pharmacokinetics of new-generation antiepileptic drugs at the extremes of age: an update. Clin Pharmacokinet. 2013;52:627-645.

4. Gambhir SS. Molecular imaging of cancer with positron emission tomography. Nat Rev Cancer. 2002;2:683-693.

5. Ishibashi K, Ishii K, Oda K, Mizusawa H, Ishiwata K. Competition between ${ }^{11} \mathrm{C}$ raclopride and endogenous dopamine in Parkinson's disease. Nucl Med Commun. 2010;31:159-166.

6. Morris ED, Yoder KK. Positron emission tomography displacement sensitivity: predicting binding potential change for positron emission tomography tracers based on their kinetic characteristics. J Cereb Blood Flow Metab. 2007;27: 606-617.

7. Paterson LM, Tyacke RJ, Nutt DJ, Knudsen GM. Measuring endogenous 5-HT release by emission tomography: promises and pitfalls. J Cereb Blood Flow Metab. 2010;30:1682-1706.

8. Jenkins PO, Mehta MA, Sharp DJ. Catecholamines and cognition after traumatic brain injury. Brain. 2016;139:2345-2371.

9. Aznar S, Hervig Mel S. The 5-HT2A serotonin receptor in executive function: Implications for neuropsychiatric and neurodegenerative diseases. Neurosci Biobehav Rev. 2016;64:63-82.

10. Berg AT, Berkovic SF, Brodie MJ, et al. Revised terminology and concepts for organization of seizures and epilepsies: report of the ILAE Commission on Classification and Terminology, 2005-2009. Epilepsia. 2010;51:676-685.

11. Van Laere K, Varrone A, Booij J, et al. EANM procedure guidelines for brain neurotransmission SPECT/PET using dopamine D2 receptor ligands, version 2. Eur J Nucl Med Mol Imaging. 2010;37:434-442.

12. Gelfand MJ, Parisi MT, Treves ST. Pediatric radiopharmaceutical administered doses: 2010 North American consensus guidelines. J Nucl Med. 2011;52: 318-322.

13. Didelot A, Mauguiere F, Redoute J, et al. Voxel-based analysis of asymmetry index maps increases the specificity of ${ }^{18} \mathrm{~F}-\mathrm{MPPF}$ PET abnormalities for localizing the epileptogenic zone in temporal lobe epilepsies. J Nucl Med. 2010;51: 1732-1739.

14. Sudha S, Lakshmana MK, Pradhan N. Chronic phenytoin induced impairment of learning and memory with associated changes in brain acetylcholine esterase activity and monoamine levels. Pharmacol Biochem Behav. 1995;52: $119-124$. 
15. Ponnusamy R, Pradhan N. The effects of chronic administration of ethosuximide on learning and memory: a behavioral and biochemical study on nonepileptic rats. Behav Pharmacol. 2006;17:573-580.

16. Mishra A, Goel RK. Comparative behavioral and neurochemical analysis of phenytoin and valproate treatment on epilepsy induced learning and memory deficit: search for add on therapy. Metab Brain Dis. 2015;30:951-958.

17. Shank RP, Maryanoff BE. Molecular pharmacodynamics, clinical therapeutics, and pharmacokinetics of topiramate. CNS Neurosci Ther. 2008;14: $120-142$.

18. Theodore WH, Giovacchini G, Bonwetsch R, et al. The effect of antiepileptic drugs on 5-HT-receptor binding measured by positron emission tomography. Epilepsia. 2006;47:499-503.

19. Hamed SA. The aspects and mechanisms of cognitive alterations in epilepsy: the role of antiepileptic medications. CNS Neurosci Ther. 2009;15:134-156.

20. Baulac M, de Boer H, Elger C, et al. Epilepsy priorities in Europe: a report of the ILAE-IBE Epilepsy Advocacy Europe Task Force. Epilepsia. 2015;56: 1687-1695.

21. Nyberg L, Karalija N, Salami A, et al. Dopamine D2 receptor availability is linked to hippocampal-caudate functional connectivity and episodic memory. Proc Natl Acad Sci USA. 2016;113:7918-7923.

22. Bortolozzi A, Diaz-Mataix L, Scorza MC, Celada P, Artigas F. The activation of 5 -HT receptors in prefrontal cortex enhances dopaminergic activity. J Neurochem. 2005;95:1597-1607.

23. Ji G, Sun H, Fu Y, et al. Cognitive impairment in pain through amygdala-driven prefrontal cortical deactivation. J Neurosci. 2010;30:5451-5464.
24. Shenhav A, Botvinick MM, Cohen JD. The expected value of control: an integrative theory of anterior cingulate cortex function. Neuron. 2013;79:217-240.

25. Helmstaedter C, Kurthen M, Lux S, Reuber M, Elger CE. Chronic epilepsy and cognition: a longitudinal study in temporal lobe epilepsy. Ann Neurol. 2003;54:425-432.

26. Appleton RE, Freeman A, Cross JH. Diagnosis and management of the epilepsies in children: a summary of the partial update of the 2012 NICE epilepsy guideline. Arch Dis Child. 2012;97:1073-1076.

27. Witt JA, Helmstaedter C. Monitoring the cognitive effects of antiepileptic pharmacotherapy: approaching the individual patient. Epilepsy Behav. 2013;26: 450-456.

28. Van Paesschen W, Dupont P, Sunaert S, Goffin K, Van Laere K. The use of SPECT and PET in routine clinical practice in epilepsy. Curr Opin Neurol. 2007;20:194-202.

29. Tripathi PP, Bozzi Y. The role of dopaminergic and serotonergic systems in neurodevelopmental disorders: a focus on epilepsy and seizure susceptibility. Bioimpacts. 2015;5:97-102.

30. Landvogt C, Buchholz HG, Bernedo V, Schreckenberger M, Werhahn KJ. Alteration of dopamine D2/D3 receptor binding in patients with juvenile myoclonic epilepsy. Epilepsia. 2010;51:1699-1706.

31. Werhahn KJ, Landvogt C, Klimpe S, et al. Decreased dopamine D2/D3-receptor binding in temporal lobe epilepsy: an $\left[{ }^{18} \mathrm{~F}\right]$ fallypride PET study. Epilepsia. 2006;47:1392-1396.

32. Guiard BP, Di Giovanni G. Central serotonin-2A (5-HT2A) receptor dysfunction in depression and epilepsy: the missing link? Front Pharmacol. 2015;6:46. 